\title{
Dynamics and Merging in Compact Galaxy Groups
}

\author{
J.-Q. Zheng, M. J. Valtonen, A. D. Chernin and S. Wiren \\ Tuorla Observatory, University of Turku, Finland
}

\begin{abstract}
.
By using a few body code with a frictional force we study the dynamics of small galaxy groups. The results have been compared with observational data on triples and binaries by Karachentsev et al. We start with four to five galaxy groups initially and take samples of the groups when the membership has dropped to two or three. It is possible to find merger evolution models which agree with observations. One interesting factor is the mass-to-light ratio of the observed systems: we find the best fit with the models when the mass-to-light ratio is about 50 in solar units.
\end{abstract}

\section{Introduction}

Compact groups of galaxies serve as a natural laboratory for studies of the formation, evolution and interaction of stellar systems. Unlike in systems of stars, merger evolution is a common phenomenon among galaxies. Interaction of galaxies is of special interest in this context because it can determine the major observed features of these systems as well as the properties of their member galaxies. The net result of merging may be the transformation of a group of several galaxies into a triplet or even to a binary, if not into a single galaxy. Interaction of galaxies in groups has been under extensive observational studies for more than three decades. A systematic catalog of 84 compact triplets of galaxies has been produced and analyzed by Karachentsev et al. (1989) on the basis of observations with 6-meter telescope of SAO (Russia). Also there is a catalog of 585 close binary galaxies (CCB) by Karachentsev $(1987,1990)$. Our numerical studies are based upon these catalogs and our results would be compared with them.

\section{Numerical method}

In our computer models, we use a traditional N-body code by Aarseth (1971). For encounter cases, we have used a frictional force calculated by the Chandrasekhar (1943) prescription. If a close encounter of two galaxies takes place, the dynamical friction is introduced, and finally, when the centers of galaxies have come within $1 \mathrm{kpc}$ of each other, the two galaxies merge into one galaxy completely (for details see Valtonen et al. 1993 and Zheng et al. 1993). 


\section{Triple Galaxies}

In order to study compact triplets, we started the simulations with systems of five galaxies which have equal masses. We integrate the orbits until the systems become triplets or until the limit of the Hubble time. We have studied 500 groups and obtamed coordinates and velocities of galaxies in each triplet. In order to compare with observational data by Karachentsev et al., we have used random projections to get separations and radial velocities of the calculated triplets.

The effect of merging reveals itself most directly in the observed statistics of their configurations. These statistics prove to be in obvious contrast with the results of computer simulations of three-body dynamics. These simulations (Chernin \& Mikkola 1991) indicate that there should be a strongly predominant three-body configuration, namely the hierarchical configuration with a close binary and a remote third body. Observational data on the compact triplets give no sign of such an excess of the hierarchical configurations (Chernin et al. 1989, 1994). Our evolutionary models can explain this contradiction (Figure 1). It appears that galaxy merging in compact groups decreases the relative number of hierarchical configurations to the observed modest level (Zheng et al. 1993). Our simulations also produce statistics in agreement with observations of the radial velocity difference and projected separations in compact triplets.

\section{Binary Galaxies}

Following the same line of argument, we study the evolutionary models of binary galaxies where merging plays a dominant role. Unlike in the case of triplets, we use a continuous distribution of galaxy masses between given upper and lower limits, and start the simulations from four galaxies in each group. We integrate the orbital motions until the system becomes a binary or until the time limit comes up. In order to compare with CCB samples in luminosity ratios, we put an up limit 8.75 for final binary mass ratio, and use a -1.5 power law for the mass distribution. We obtain distributions of the separations, radial velocities and masses of the binaries. The distribution of separations $\left(R_{\text {pro }}\right)$ agree with observations reasonably well. We also have a good agreement in radial velocity between simulations and observations.

By comparing the observed luminosity distribution and the calculated mass distribution, we obtain $50 \pm 15$ solar units for the mass-to-light ratio (Hubble constant $\left.H_{0}=75 \mathrm{~km} \mathrm{~s}^{-1} \mathrm{Mpc}^{-1}\right)$. This value is the same also in the triplets result (Zheng et al. 1993). The two distributions do not match perfectly - the major difference is in the range of very small masses (Figure 2).

\section{Discussion}

The usual methods of analyzing triple galaxy and binary galaxy data include many assumptions about the orbital distributions of the galaxies. For example, the assumption about the eccentricities in binary galaxies has a strong influence on the derived mass-to-light ratio. Also the results are sensitive to the adopted cut-off in the projected distance and in the radial velocity. In the present method the orbital distributions evolve "naturally" via galaxy encoun- 


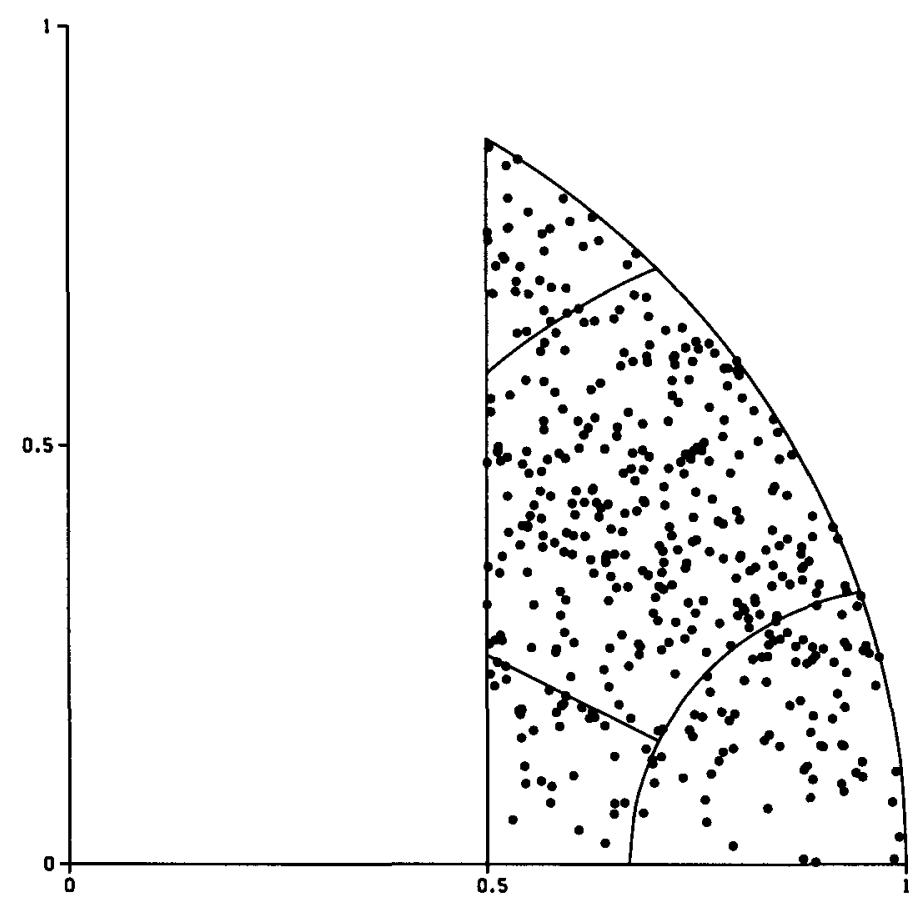

Figure 1. Triplets configurations in the simulated results. One of the galaxies is at the coordinate origin, the second one is at the point $(1,0)$ while the third one is shown by a dot. This representation is called the Agekian-Anosova map of three-body configurations. 


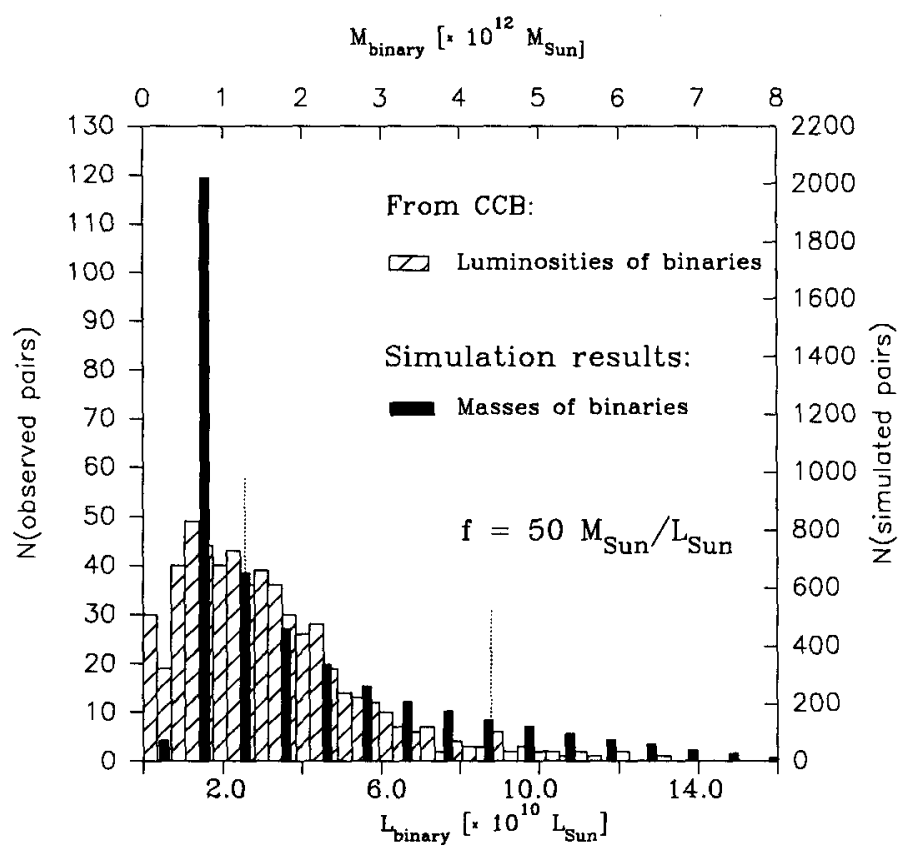

Figure 2. The overlay of the mass distribution of binaries in the simulations (scale on top of the diagram and on the right) and the luminosity distribution of the observed CCB binaries (bottom scale and the left hand scale). The overlay assumes the mass-to-light ratio of 50 in solar units. 
ters and mergers. Also the distributions as a whole are used in the comparison between observations and theory rather than mean values. Therefore there is no need to introduce cut-off values in the observational samples. As long as the real evolution of triples and binaries have followed even somewhat similar paths as the simulations, we obtain very reliable values for the mass-to-light ratios in galaxies in small groups. Both for binaries and triplets this value is not far from 50 in solar units.

\section{References}

Aarseth, S. J. 1971, Ap \& SS, 14, 20

Chernin, A. D., Ivanov, A. V. \& Trofimov, A. V. 1989, Astron. Circ. (USSR), NO. 1540,3

Chernin, A. D., Ivanov, A. V., Trofimov, A. V. \& Mikkola, S. 1994, A \& A, 281, 685

Chernin, A. D. \& Mikkola, S. 1991, MNRAS, 253, 153

Chandrasekhar, S. 1943, ApJ, 97, 255

Karachentsev, I. D. 1987, Binary Galaxies, Moscow, Nauka

Karachentsev, I. D. 1990, in Paired and Interacting Galaxies, IAU Colloquium No. 124, edited by J. W. Sulentic et al., NASA Conf. Publ. 3098, p. 3

Karachentsev, I. D., Karachentseva, V. E. \& Lebedev, V. S., Izv. Spec. Astrophys. Obs., 27, 67

Valtonen, M. J., Byrd, G. G., McCall, M. L. \& Innanen, K. A. 1993, AJ, 105, 886

Zheng, J.-Q., Valtonen, M. J. \& Chernin, A. D. 1993, AJ, 105, 2047 